\title{
Ampliación de la distribución geográfica y estado de conservación de Ctenoblepharys adspersa (Tschudi, 1845)
}

\author{
Geographical range extension and conservation status of Ctenoblepharys adspersa \\ (Tschudi, 1845)
}

\author{
Jesús R. Ormeño*1, 2,3,8 \\ https://orcid.org/0000-0002-7324-0561 \\ ormexo_benavides@hotmail.com \\ Ronal Sumiano-Mejia ${ }^{2,4}$ \\ https://orcid.org/0000-0001-6193-3574 \\ ronalbio_1504@outlook.com \\ Alfonso Orellana-Garcia ${ }^{2,4,5}$ \\ https://orcid.org/0000-0003-4021-695X \\ alfonso.orellana@unmsm.edu.pe \\ Oliver Q. Whaley ${ }^{2,6}$ \\ o.whaley@kew.org \\ José Pérez Z. 5, 7, 8 \\ https://orcid.org/0000-0003-2756-815 \\ jose.perez.z@upch.pe \\ Pablo J. Venegas ${ }^{1,3}$ \\ https://orcid.org/0000-0002-6501-4492 \\ sancarranca@yahoo.es
}

\section{*Corresponding author}

1 División de Herpetología. Centro de Ornitología y Biodiversidad (CORBIDI). Lima, Perú.

2 Huarango Nature - Conservamos Ica (CÓNICA). Ica, Perú.

3 Instituto Peruano de Herpetología (IPH), Lima, Perú.

4 Facultad de Ciencias Biológicas, Universidad Nacional San Luis Gonzaga. Ica, Perú.

5 Museo de Historia Natural. Universidad Nacional Mayor de San Marcos. Lima, Perú.

6 Royal Botanic Gardens, Kew. Richmond, Surrey, TW9 3AE, UK.

7 Laboratorio de Estudios en Biodiversidad (LEB), Laboratorios de Investigación y Desarrollo, Universidad Peruana Cayetano Heredia. Lima, Perú.

8 Laboratorio de Ecología Integrativa, Facultad de Ciencias Biológicas, Universidad Nacional Mayor de San Marcos. Lima, Perú.

\section{Citación}

Ormeño JR, Sumiano-Mejia R, Orellana-Garcia A, Whaley O, Pérez J, Venegas PJ. 2021. Ampliación de la distribución geográfica y estado de conservación de Ctenoblepharys adspersa (Tschudi, 1845). Revista peruana de biología 28(3):e21118 (Agosto 2021). doi: http://dx.doi.org/10.15381/rpb. v28i3.21118

$\begin{array}{ll}\text { Presentado: } & 13 / 07 / 2020 \\ \text { Aceptado: } & 27 / 02 / 2021\end{array}$

Publicado online: $30 / 08 / 2021$

Editor: César Aguilar Puntriano

\section{Resumen}

Ampliamos la distribución geográfica de Ctenoblepharys adspersa (Liolaemidae), una especie de saurio endémico y amenazado del desierto peruano. Nuestro nuevo registro extiende la distribucion de esta especie en $60 \mathrm{~km}$ (línea recta) de la localidad más oriental previamente conocida, la Reserva Nacional de Paracas. Registramos dos tipos de hábitat nuevos para $C$. adspersa al interior de las estribaciones andinas e identificamos las plantas nativas asociadas a sus hábitats. Además, revisamos el estado de conservación de esta especie y los desafíos que implican su conservación, resaltando que la mayoría de las poblaciones son vulnerables a los impactos en su hábitat producto del desarrollo de infraestructura urbana y/o agrícola.

\section{Abstract}

We extend the geographical distribution of Ctenoblepharys adspersa (Liolaemidae) an endemic and threatened lizard species from the Peruvian desert. Our new record extends the known species distribution ca. $60 \mathrm{~km}$ (straight line) east-southeastern from the eastemost record at Paracas National Reserve. We recorded two new type of habitat for $C$. adspersa that reach to the Pacific foothills and identified the native plants associated to its habitats. Moreover, we review the conservation status and the challenges that facing it, highlighted that most of its populations are vulnerable to the impacts on their habitat caused by the development of urban and agricultural infrastructure.

Palabras claves:

Liolaemidae; hábitats; historia natural; desierto; lagartija cabezona; especie endémica.

Keywords:

Liolaemidae; habitats; natural history; desert; big-headed lizard; endemic species.

\section{Introducción}

La Familia Liolaemidae es la más diversa de la zona sur de Sudamérica, y se encuentra integrada por los géneros Liolaemus, Phymaturus y Ctenoblepharys (Lobo et al. 2012, Abdala \& Quinteros 2014, Ormeño et al. 2021, Abdala et al. 2021). El género Ctenoblepharys actualmente es considerado monoespecífico por Laurent (1984), después de haber tenido una larga y complicada historia taxonómica, con 12 especies incluidas en este género que luego fueron transferidas a Liolaemus y Phymaturus (Etheridge 1995). El holotipo de C. adspersa fue descrito por Tschudi (1845) con localidad tipo en la Hacienda Acaray, a 2 leguas (aproximadamente $10 \mathrm{~km})$ de Huacho $\left(11^{\circ} 07^{\prime} \mathrm{S}, 77^{\circ} 37^{\prime} \mathrm{W}\right.$, Lima, Perú) (Nuñez \& Yañez 1983, Tello 1998), y se encuentra depositado en la colección del Muséum d'Histoire Naturelle de Neuchâtel, Suiza (Donoso-Barros 1971). Asimismo, la evidencia molecular indica que el origen de Ctenoblepharys adspersa se habría producido en el cretáceo superior hace 77 millones de años (Aguilar et al. 2018). 
Ctenoblepharys adspersa, es una especie endémica del desierto costero central sur peruano (Etheridge 1995, Tello 1998, Abdala \& Quinteros 2014), con una distribución restringida entre Lima y Arequipa desde los 0 a 800 m de altitud (Vargas 2015, Aguilar et al. 2017, Pérez 2018). Este saurio se caracteriza por tener una baja amplitud de nicho espacial, con preferencias por hábitats desérticos de escasa o sin vegetación, como dunas (Etheridge 1995, Pérez \& Balta 2007, Vargas 2015), lomas y tilandsiales (Aguilar et al. 2019). Además presenta hábitos diurnos, principalmente en horarios con poca radiación solar, generalmente a inicios de la mañana y final de la tarde (Pérez \& Balta 2007, Vargas 2015). La dieta de $C$. adspersa se encuentra restringida a insectos, con predominio de himenópteros, seguido de coleópteros y larvas de insectos (Pérez \& Balta 2007).

La única investigación referente a $C$. adspersa fue realizada en las poblaciones que habitan la Reserva Nacional de Paracas (Pérez \& Balta 2007); asimismo, se conoce que es una especie poco frecuente, con una distribución limitada a $18000 \mathrm{~km}^{2}$, como resultado de la pérdida y fragmentación de sus hábitats (Pérez 2018). Por lo tanto, se encuentra categorizada como Vulnerable en la Lista Roja de la Unión Internacional para la Conservación de la Naturaleza.

Debido a los vacíos de información o limitado conocimiento de $C$. adspersa, esta investigación tiene como objetivo actualizar su distribución geográfica, reportar nuevas localidades, uso de hábitats y destacar aspectos de su historia natural, esperando contribuir en el entendimiento de su biología y conservación.

\section{Materiales y métodos}

Las evaluaciones de campo fueron realizadas entre el 2015-2020, en un distrito del departamento de Lima: Huacho; seis distritos del departamento de Ica: Los Aquijes, Pueblo Nuevo, Yauca del Rosario, Santiago (Provincia de Ica), Paracas (Provincia de Pisco) y San Juan de Marcona (Provincia de Nasca); y dos distritos del departamento de Arequipa: Lomas y Bella Unión. Estas evaluaciones fueron realizadas como parte del Proyecto Huarango Nature (CÓNICA), donde se colectó flora y fauna silvestre con fines de investigación científica.

Se realizaron búsquedas exhaustivas en horario diurno entre las 8:00 y 16:00 hrs., donde se ha reportado actividad de la especie (Pérez \& Balta 2007). Se colectaron especímenes, siguiendo una metodología estandarizada de captura y preservación (Barry 2012). Los especímenes colectados fueron depositados en el Departamento de Herpetología del Museo de Historia Natural de la Universidad Nacional Mayor de San Marcos (MUSM) y en la División de Herpetología del Centro de Ornitología y Biodiversidad (CORBIDI). Además, se llevo a cabo la revisión de especímenes en colecciones científicas (ver especímenes examinados en el Anexo 1). Las identificaciones fueron realizadas siguiendo la descripción general y caracteres morfológicos según lo descrito por Tschudi (1845) (Fig. 1) y Etheridge (1995).

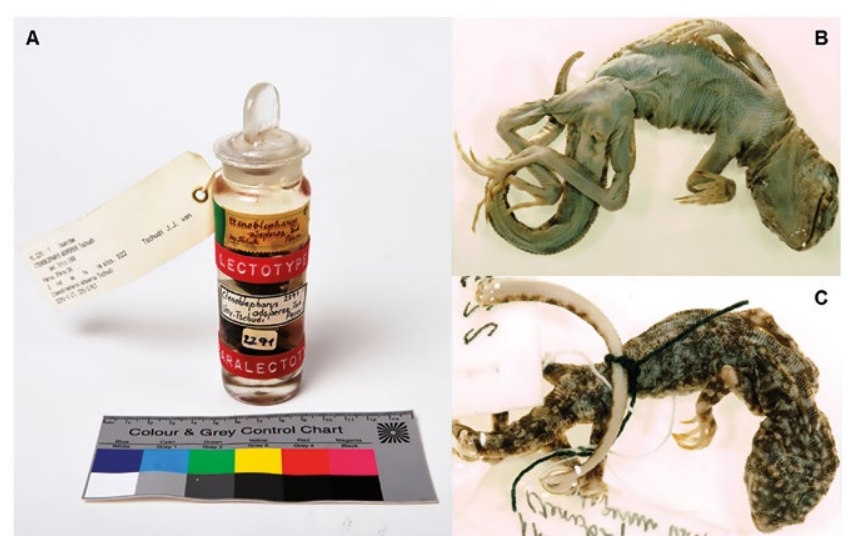

Figura 1: Especímenes Tipo de Ctenoblepharys adspersa en el Natural History Museum Neuchâtel (Switzerland) - MHNN. (Izquierda) Tipo: Type, 2 ind., ma fe, Pérou [91.2291 T]; (Derecha/ Arriba) Lectotipo: Macho, adulto, posición ventral [MHNN 2291-1] y (Derecha/Abajo) Paralectotipo: Hembra, adulto, posición dorsal [MHNN 2291-2]. Fotos: (Izquierda): CCelia Bueno-MHNN y (Derecha): (CAlessandro Catenazzi.

Para el análisis de estado de conservación se utilizó el programa ArcGIS versión 10.6 (ESRI 2018) además del software en línea GeoCAT (Bachman et al. 2011), considerando los tamaños de cuadrícula $(2 \times 2 \mathrm{~km})$ sugeridos por los criterios de la Lista Roja de la IUCN Versión 3.1. Segunda edición (IUCN 2012).

La toma de datos de flora se realizó por observación directa y colectas in situ. La colección y herborización se realizaron siguiendo las técnicas estandarizadas según (Bridson \& Forman 1992, Arakaki 2001). Los especímenes fueron depositados en el Herbario San Marcos (USM) y Royal Botanical Garden Kew (K). Para la determinación botánica, se revisó literatura especializada (MacBride 1943, Tovar 1993, Brako \& Zarucchi 1993, Whaley et al. 2010, 2019, Ostolaza 2019); el tratamiento de las familias botánicas se basó en la clasificación taxonómica The Angiosperm Phylogeny Group IV (APG IV 2016). Asimismo, la clasificación y descripción de los hábitats se complementó con la guía de plantas y vegetación de Ica (Whaley et al. 2010), la lista anotada de flora y hábitats para Ica (Whaley et al. 2019) y el mapa nacional de ecosistemas del Perú (MINAM 2018).

\section{Resultados}

Distribución.- Se registraron un total de siete nuevas localidades para Ctenoblepharys adspersa, encontrándose una localidad en el departamento de Lima: Reserva Nacional de Lachay, cinco localidades en el departamento de Ica: Área de Conservación Regional Laguna de Huacachina (ACRLH), Lomas de Marcona, Lomas de Amara-Ullujaya, Cerro Riachuelo y Quebrada Cocharcas. Esta última representa el registro más oriental (apróximadamente $60 \mathrm{~km}$ de distancia de la localidad previamente conocida más próxima en la Reserva Nacional de Paracas); asimismo, se reporta una última localidad en el departamento de Arequipa: Jardín Sacaco. Estas nuevas localidades, especialmente la Quebrada Cocharcas, aumentan considerablemente su área de extensión hacia las estribaciones andinas (Fig. 2). A partir de estos nue- 
vos registros y los reportados en investigaciones anteriores, la distribución actual de Ctenoblepharys adspersa se encuentra localizada en la franja del Desierto Costero e incluye los departamentos de Lima, Ica y Arequipa.

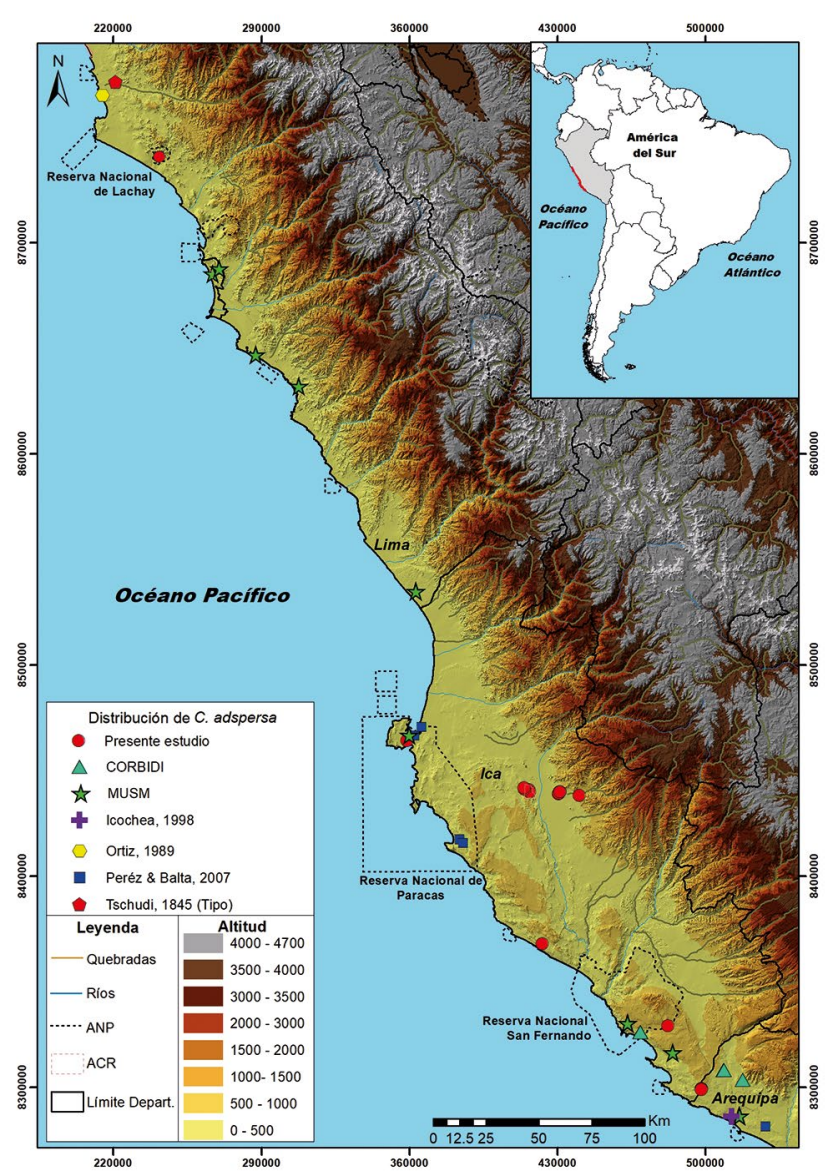

Figura 2. Distribución de Ctenoblepharys adspersa (Tschudi, 1845) dentro y fuera de ANP. Se muestran los registros empleados en el presente estudio, incluyendo especímenes de las colecciones científicas del Museo de Historia Natural (MUSM) y del Centro de Ornitología y Biodiversidad (CORBIDI).

Actualmente $C$. adspersa ha sido registrada en 26 localidades, 8 localidades para el departamento de Lima: Acaray, Cerro Lomo de Corvina, Cerro Los Perros, entre Ventanilla y Puente Piedras, Hato Viejo (Reserva Nacional de Lachay), Huacho (localidad tipo), Nuevo Cañete y Quebrada Cruz de hueso; 14 localidades en el departamento de Ica: Cerro Barlovento, Cerro Santa María, Museo de Sitio Julio C. Tello, Cabezas Largas (Reserva Nacional de Paracas), Cerro Olleros (Lomas Amara), Cerro Riachuelo, Cerros San Fernando, Quebrada Cocharcas (Río Rosario), Dunas de Huacachina, Médanos Orovilca (ACRLH), El Chaco, Lomas Marcona, Cerro Lechuza, Tilandsial Marcona (Reserva Nacional San Fernando); y 4 localidades para el departamento de Arequipa: Acarí, Aguada de Lomas, Arenal de Bella Unión y Jardín Sacaco. Cabe resaltar que el registro fotográfico en Huarmey del departamento de Áncash (Aguilar et al. 2017) no se consideró para este reporte debido a la falta de un espécimen testigo (voucher).
Hábitats.- La coloración de Ctenoblepharys adspersa es una perfecta adaptación a sus hábitats desérticos, ya que la coloración dorsal del cuerpo imita los granos y colores de sustratos arenosos (Fig. 3).
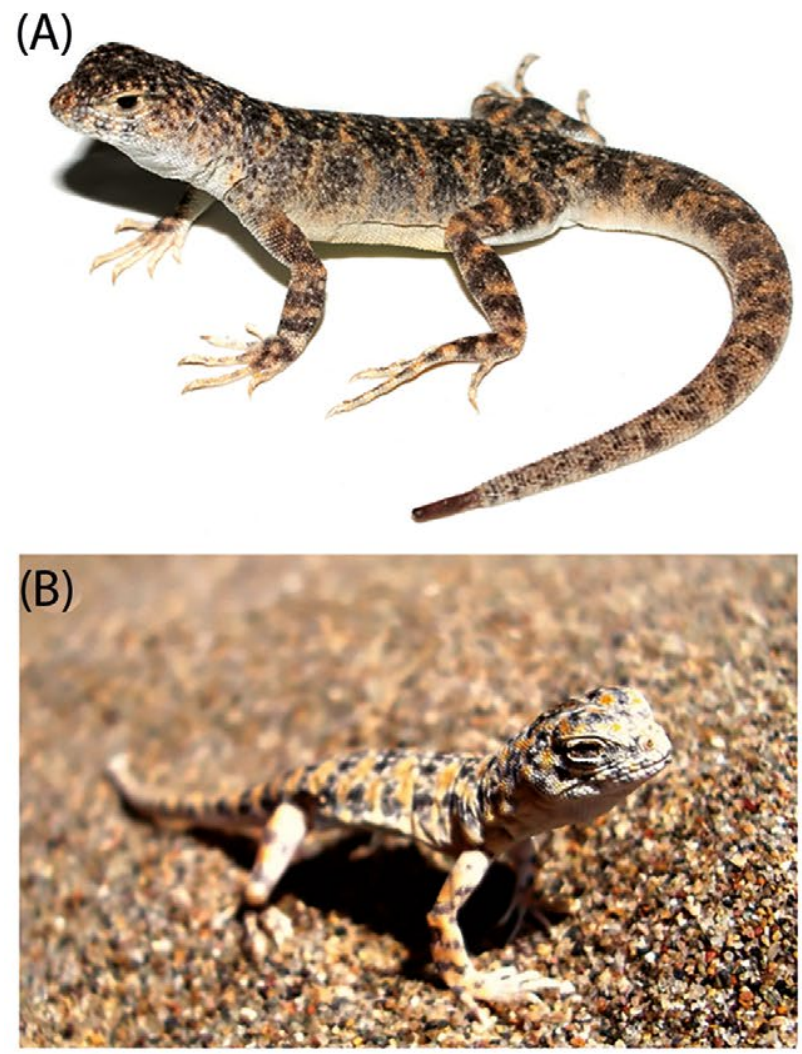

(C)

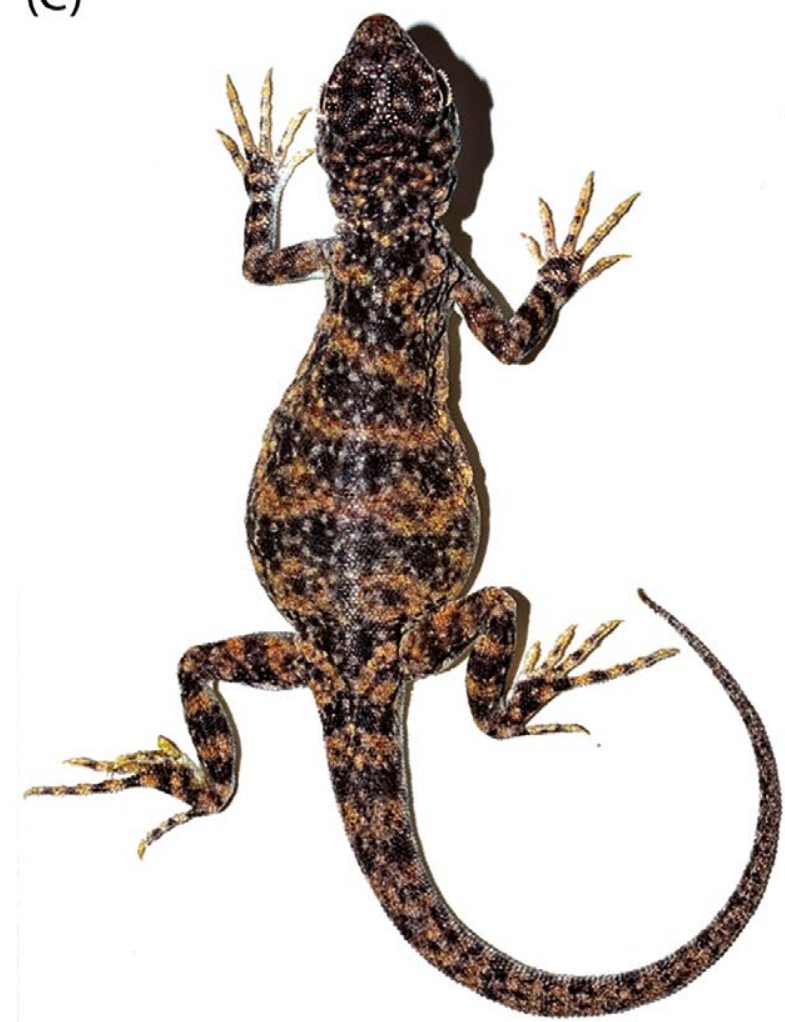

Figura 3: Ctenoblepharys adspersa: (A) macho adulto, (B) Juvenil en hábitat; y (C) hembra adulta preñada. Fotos: Jesús Ormeño (A y C) y Alfonso Orellana (B). 
Los individuos observados de $C$. adspersa estuvieron empleando cinco tipos de hábitats como: desierto, tilandsial en duna, tilandsial en quebrada, lomas y huaycos (escorrentías o arroyos efimerales). Cabe indicar que el tilandsial en quebrada y la vegetación de los huaycos efímeros son nuevos hábitats reportados para este saurio. El hábitat de desierto donde fue registrado C. adspersa está caracterizado por la presencia de dunas o planicies arenosas con escasa vegetación. No obstante, para el caso del ACRLH se observaron algunos parches de Tiquilia dichotoma (Boraginaceae) especie nodriza, importante para $C$. adspersa debido a que brinda refugio $\mathrm{y}$ alimento como atrayente de insectos.

El hábitat de tilandsial en duna presentó una vegetación casi homogénea conformada por especies de Tillandsia spp., hierbas efimerales de Solanaceae (Solanum edmonstonei), Oxalidaceae (Oxalis spp.), Poaceae (Eragrostis spp.) y Cactaceae (Haageocereus decumbens) que se encuentran sobre un sustrato arenoso fino. El tilandsial en quebrada, ubicado al oriente de la línea marinocostera e inicio de las estribaciones andinas (quebradas), presenta una vegetación más heterogénea dominada por
Bromeliaceae (Tillandsia latifolia), Cactaceae columnares (Armatocereus procerus, Neoraimondia arequipensis y Haageocereus spp.) y matorrales xerofíticos, como Zygophyllaceae (Bulnesia retama), que se encuentran sobre un sustrato arenoso y zonas rocosas. La loma (oasis de niebla) presenta vegetación heterogénea, con especies efimerales como Boraginaceae (Tiquilia spp.), Solanaceae (Nolana spp.), Poaceae (Stipa pachypus), Malvaceae (Palaua spp.), Fabaceae (Hoffmannseggia spp. y Weberbauerella raimondiana); algunas bulbosas, perennes como Asteraceae (Ambrosia dentata), Amaranthaceae (Atriplex rotundifolia) y Cactaceae (Haageocereus decumbens, Islaya islayensis), musgos y líquenes. Los huaycos corresponden a las zonas de vegetación transitoria, bajada de escorrentías o arroyos efimerales, presentando hierbas estacionales como Fabaceae (Hoffmannseggia viscosa), Plantaginaceae (Galvezia fruticosa), Asteraceae (Encelia canescens) y matorrales perennes de Zygophyllaceae (Bulnesia retama), Fabaceae (Parkinsonia praecox); y árboles de porte arbustivo como Capparaceae (Capparis avicennifolia) que se encuentran sobre sustrato de textura arcillosa-limosa y arenosa (Fig. 4).

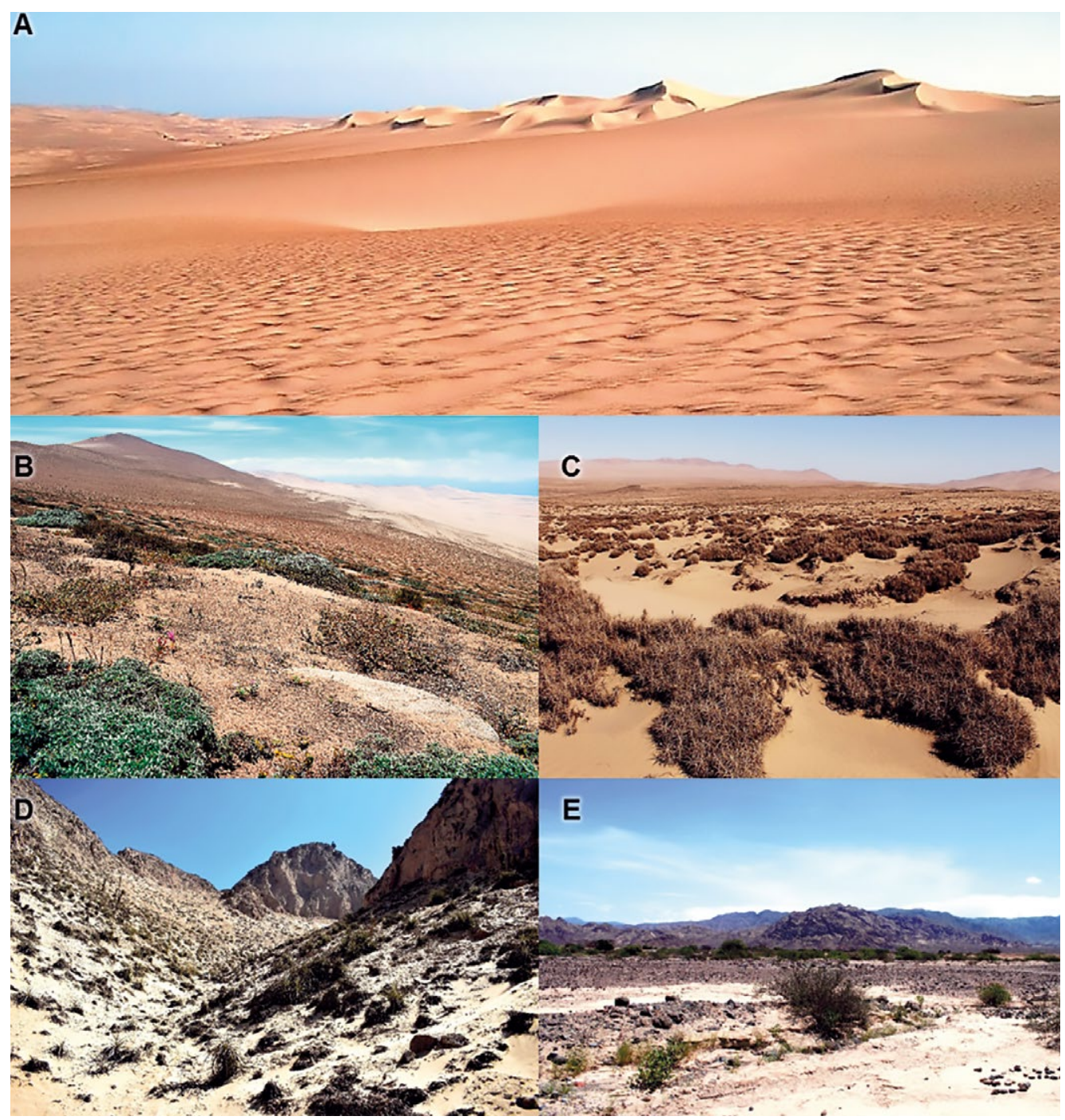

Figura 4: Hábitats de Ctenoblepharys adspersa: A) Desierto (dunas), B) Lomas costeras, C) Tilandsial en dunas, D) Tilandsial en quebrada y E) Huayco (escorrentías o arroyos efimerales). Fotos: Oliver Whaley (A), Jesús Ormeño (B y D), Ronal Sumiano (C) y Christian Padilla (E). 
Estado de conservación.- El 37\% de registros de $C$. adspersa se encuentran dentro de las Áreas Naturales Protegidas (ANP o ACR): Reserva Nacional de Lachay, Reserva Nacional de Paracas, Reserva Nacional San Fernando y Área de Conservación Regional Laguna de Huacachina, los cuales ofrecen cierto nivel de protección por las medidas de control y gestión que presentan; asimismo, un registro (2\%) se encuentra en el ecosistema frágil Lomas de Amara (SERFOR 2018). El 61\% restante de registros de $C$. adspersa se encuentran fuera de cualquier tipo o modalidad de conservación. (Fig. 2).

La última categorización nacional de fauna silvestre considera a C. adspersa como Vulnerable (VU) B1ab (ii,iv) (Pérez 2018), con una extensión de presencia (EOO) de $18000 \mathrm{~km}^{2}$. Los nuevos registros reportados en este artículo extenderían su EOO a $20978 \mathrm{~km}^{2}$, y a partir de la totalidad de los registros compilados, se estima que su área de ocupación (AOO) sería aproximadamente de $120 \mathrm{~km}^{2}$. Tomando en cuenta los datos recopilados y actualizados en este trabajo, y los EOO y AOO mencionados, podríamos considerar que las potenciales categorías de conservación para $C$. adspersa serían Casi Amenazado (NT), considerando el EOO reportado, y En Peligro (EN) considerando el AOO estimado. Sin embargo, debido a la baja densidad y poca detectabilidad de este saurio, es probable que se puedan obtener registros adicionales dentro de su área de distribución que incrementen su AOO.

Por otro lado, las principales amenazas identificadas durante las evaluaciones de campo para $C$. adspersa fueron la intensa fragmentación de los ambientes naturales, debido al crecimiento de zonas urbanas y agrícolas, proyectos mineros formales e informales, turismo no controlado presente en diferentes zonas del desierto costero, incluida la RNP y en el ACRLH. Todas estas actividades antrópicas están ocasionando la pérdida de hábitat de esta especie y otros saurios costeros.

\section{Discusión}

Desde la descripción de $C$. adpersa se conoce que su distribución se encontraba cerca de la línea costera y solamente utilizaba el hábitat de desierto costero como dunas, playas arenosas (Etheridge 1995, Pérez \& Balta 2007, Vargas 2015, Aguilar et al. 2017, Pérez 2018) lomas y tilandsiales (Aguilar et al. 2019). No obstante, en el presente artículo ampliamos su presencia a los hábitats de tilandsial en quebradas y huayco efimerales, evidenciando una mayor amplitud en su nicho espacial, hacia áreas con mayor presencia de vegetación en la base de estribaciones andinas. Las nuevas localidades de registro representarían poblaciones más propensas hacia amenazas antrópicas, debido a que se encuentran fuera de Áreas Naturales Protegidas (ANP) o alguna modalidad de conservación.

Por otro lado, estos registros permiten incrementar el EOO en $20.978 \mathrm{~km}^{2}$, de lo reportado por Pérez (2018). Este estimado excluiría a $C$. adspersa de alguna categoría de amenaza. No obstante, el AOO que no fue considerado anteriormente para el análisis de categorización de la especie, en la actualidad se estimaria en $120 \mathrm{Km}^{2}$.
Considerando que el hábitat disponible para $C$. adspersa está subestimado y reducido por los impactos ya mencionados, deben priorizarse los estudios de escala espacial de los hábitats disponibles, poblaciones, localidades y amenazas; con la finalidad de reevaluar su estatus de conservación. Por lo tanto, consideramos recomendable mantener la categoría de Vulnerable (VU) para esta especie endémica.

\section{Literatura citada}

Abdala CS \& Quinteros AS. 2014. Los últimos 30 años de estudios de la familia de lagartijas más diversa de Argentina: Actualización taxonómica y sistemática de Liolaemidae. Cuadernos de Herpetología 28 (2): 55-82.

Abdala CS, Laspiur A \& Langstroth RP. 2021. Las especies del género Liolaemus (Liolaemidae). Lista de taxones y comentarios sobre los cambios taxonómicos más recientes. Cuadernos de Herpetología 35(3): 193-222.

Aguilar C, Quiroz Rodriguez A, Perez J, Catenazzi A, Lundberg M. 2017. Ctenoblepharys adspersa. The IUCN Red List of Threatened Species 2017: e.T169714A48671559. https://dx.doi.org/10.2305/IUCN.UK.2017-2.RLTS. T169714A48671559.en

Aguilar-Puntriano C, Ávila LJ, De la Riva I, Johnson L, Morando M, Troncoso-Palacios J, Wood PL, Sites JW. 2018. The shadow of the past: convergence of young and old South American desert lizards as measured by head shape traits. Ecology and Evolution 2018 (8): 1139911409. https://doi.org/10.1002/ece3.4548.

Aguilar-Puntriano C, Ramírez C, Castillo E, Mendoza A, Vargas VJ, Sites J. W. 2019. Three new lizard species of the Liolaemus montanus group from Perú. Diversity, 11(9): 161. https://doi.org/10.3390/d11090161.

APG IV (Angiosperm Phylogeny Group). 2016. An update of the Angiosperm Phylogeny Group classification for the orders and families of flowering plants: APG IV. Botanical Journal of the Linnean Society 181:1-20. https:// doi.org/10.1111/boj.12385

Arakaki M. 2001. Herbario de cactáceas. Quepo, 15, 38-45.

Bachman S, Moat J, Hill AW, et al. 2011. Supporting Red List threat assessments with GeoCAT: geospatial conservation assessment tool. ZooKeys 150: 117-126. https://doi.org/10.3897/zookeys.150.2109.

Barry SJ. 2012. Preparing scientific specimens. Pp. 96 - 105. En: McDiarmid, R. W., M. S. Foster, C. Guyer , J. W. Gibbons y N. Chernoff (eds.). Reptile Biodiversity: Standard Methods for Inventory and Monitoring. University of California Press, Berkeley Los Angeles London, 424 pp. https://doi.org/10.1111/j.1095-8312.2012.01975.x

Brako L \& Zarucchi J. 1993. Catalogue of the Flowering Plants and Gymnospermas of Perú. Monographs in Systematic Botany from the Missouri Botanical Garden 45: 1 $-1286$.

Bridson D \& Forman L. 1992. The Herbarium Handbook. Great Britain. Royal Botanic Gardens, Kew.

Donoso-Barros R. 1971. The genera Ctenoblepharis and Phrynosaura. Herpetological Review 3(5): 85.

ESRI (Environmental Systems Resource Institute). 2018. ArcMap 10.6. - ESRI, Redlands, California.

Etheridge R. 1995. Redescription of Ctenoblepharys adspersa Tschudi, 1845, and the taxonomy of Liolaeminae (Reptilia, Squamata, Tropiduridae). American Museum Novitates $\mathrm{N}^{\circ} 3142$. 
IUCN (International Union for Conservation of Nature). 2012. IUCN Red List Categories and Criteria: Version 3.1. Second edition. (Gland, Switzerland and Cambridge, UK: IUCN). VI 34pp. Available from: <http://www.iucnredlist.org $>$. Acceso: 02/02/2020.

Laurent RF. 1984. On some iguanid genera related to or previously confused with Liolaemus Wiegmann. Journal of Herpetology: 357-373. https://doi. org/10.2307/1564098

Lobo F, Abdala C \& Valdecantos S. 2012. Morphological diversity and phylogenetic relationships within a SouthAmerican clade of iguanian lizards (Liolaemidae: Phymaturus). Zootaxa 3315(1): 1-41. https://doi. org/10.11646/zootaxa.3315.1.1

Macbride JF. 1943. Leguminosae, Flora of Peru. Field Museum of Natural History, Chicago, Botanical Series 13 (3/1): 3-507.

MINAM (Ministerio del Ambiente). 2018. Mapa Nacional de Ecosistemas del Perú: Memoria descriptiva. Lima, Perú. 118 pp.

Nuñez H \& Yañez J. 1983. Abas y Velos aura nuevos géneros de lagartos Iguanidae y proposiciones sistemáticas respecto de los géneros aliados (Reptilia: Squamata). Boletín-Museo Nacional de Historia Natural 40: 97-105.

Ormeño R, Sumiano-Mejia R, Orellana-García A, et al. 2021. Ampliación de la distribución geográfica de Liolaemus nazca Aguilar, Ramírez, Castillo, Mendoza, Vargas \& Sites Jr., 2019 (Iguania: Liolaemidae) para el extremo sur de Ica y norte de Arequipa, Perú-Hábitats y conservación. Cuadernos de Herpetología, 35(3): 237-244.

Ostolaza C. 2019. Todos los cactus del Perú. 2da Edición. Sociedad Peruana de Cactáceas y Suculentas. Lima. 572 pp.

Pérez J \& Balta K. 2007. Ecología de la comunidad de saurios diurnos de la Reserva Nacional de Paracas, Ica, Perú. Revista Peruana de Biología 13(3): 169 - 176. https:// doi.org/10.15381/rpb.v13i3.2334.

Pérez J. 2018. Ctenoblepharys adspersa Tschudi, 1845. Pp. 464. En: SERFOR. Libro Rojo de la Fauna Silvestre Amenazada del Perú. Primera edición. Servicio Nacional Forestal y de Fauna Silvestre. Lima, Perú. 548 pp.

SERFOR (Servicio Nacional Forestal y de Fauna Silvestre). 2018. Resolución de Dirección Ejecutiva que aprueba la incorporación de los treinta y seis (36) ecosistemas, que como Anexo forma parte integrante de la presente Resolución, a la "Lista Sectorial de Ecosistemas Frágiles", aperturada mediante Resolución Ministerial $\mathrm{N}^{\circ}$ 0274-2013-MINAGRI. El Peruano 1671611-1: 34-35.

Tello G. 1998. Lagartijas del Departamento de Lima, Perú. Biotempo 3: 57-61. https://doi.org/10.31381/biotempo. v3i0.1528.
Tovar O. 1993. Las gramíneas (Poaceae) del Perú. Madrid. Ruizia 13: 1-481.

Tschudi JJ. 1845. Reptilium conspectum quae in republica peruana reperiuntur er pleraque observata vel collecta sunt in itenere. Archiv für Naturgeschichte 11 (1): 150-170. https://doi.org/10.5962/bhl.part.7963.

Vargas V. 2015. Guía de Identificación de anfibios y reptiles. PERU LNG (ed). Lima. 111 pp.

Whaley O, Orellana A, Pérez E, et al. 2010 . Plantas y vegetación de Ica, Perú. Un Recurso para su restauración y conservación. Royal Botanic Gardens, Kew. 94 pp.

Whaley OQ, Orellana-Garcia A. \& Pecho JO. 2019. An Annotated Checklist to Vascular Flora of the Ica Region, Peruwith notes on endemic species, habitat, climate and agrobiodiversity. Phytotaxa 389(1): 1-125. https:// doi.org/10.11646/phytotaxa.389.1.1

\section{Agradecimientos / Acknowledgments:}

A César Aguilar del Departamento de Herpetología, Museo de Historia Natural de la UNMSM; y al Centro de Ornitología y Biodiversidad (CORBIDI) por el acceso y revisión de los especímenes. A Celia Bueno del Museo de Historia Natural de Neuchâtel (MHNN) por la información y fotografías de los ejemplares tipo; asimismo a Alessandro Catenazzi por proporcionarnos fotos de los lectotipos. Al Dr. Justin Moat (Royal Botanic Gardens, Kew) por el soporte e interpretación del GeoCAT. Al Área de Conservación Regional Laguna de Huacachina - ACRLH, por el apoyo logístico y facilidad de ingreso. A Alberto Benavides de SAMACA Orgánico por todo el apoyo logístico en salidas de campo. A los integrantes del Proyecto Huarango Nature (CÓNICA), en especial a Darwin García y lomar Santana por su colaboración en las evaluaciones de campo. A dos revisores anónimos por la sugerencias a esta Nota Científica.

\section{Conflicto de intereses / Competing interests:}

Los autores no incurren en conflictos de intereses.

\section{Rol de los autores / Authors Roles:}

JRO, RSM, AOG, OW, JP, PV: Conceptualización, Validación, Investigación, Recursos, Curación de datos, Escritura- Preparación del borrador original, Redacción: revisión y edición, JRO, RSM, AOG, OW: Adquisición de fondos. JRO, AOG, JP, PV: Supervisión. JRO, AOG: Metodología, Administración de proyecto. JRO, JP: Visualización

\section{Fuentes de financiamiento / Funding:}

Royal Botanic Gardens, Kew y J Sainsbury's plc (UK), Proyecto Huarango.

\section{Aspectos éticos / legales; Ethics / legals:}

Este trabajo involucró la colecta permanente y preservación de individuos como especímenes testigo. Colectándose no más de tres especímenes por localidad. Tal como se encentra estipulado en las autorizaciones para investigación emitidas por el Servicio Nacional Forestal y de Fauna Silvestre (SERFOR) mediante las Resoluciones de Dirección General: Nº 471-2017-SERFOR/DGGSPFFS, N 473-2017-SERFOR-DGGSPFFS, No 441-2018-MINAGRISERFOR-DGGSPFFS; por la Administración Técnica Forestal y de Fauna Silvestre de Ica (ATFFS): Nº 041-2016-SERFOR-ATFFS ICA; y por el Servicio Nacional de Áreas Naturales Protegidas por el Estado (SERNANP): Resolución Jefatural N030-2014-SERNANPRNP/J y N001-2015-SERNANP-JEF.

Apendice 1: Especímenes revisados de Ctenoblepharys adspersa (Tschudi, 1845). Colectas ordenadas por Departamento. Provincia: Distrito (Código de colección científica).

Arequipa. Caravelí: Bella Unión (CORBIDI 12007); Lomas (CORBIDI 10847), (MUSM 16170), (MUSM 16584), (CORBIDI 10848).

Ica. Nasca: San Juan de Marcona (CORBIDI 17635), (CORBIDI 17638), (CORBIDI 18880), (CORBIDI 22658*), (MUSM 16099), (MUSM 31530), (MUSM 31531), (MUSM 31532), (MUSM 40004*), (MUSM 40010*). Pisco: Paracas (MUSM 28501), (MUSM 30345). Ica: Ica (CORBIDI 22639*); Pueblo Nuevo (MUSM-40007*). Lima. Cañete: San Vicente (MUSM 28710), (MUSM 28711). Lima: Villa El Salvador (MUSM-1829), (MUSM 1835); Ventanilla (MUSM 1831), (MUSM 4670); Punta Negra (MUSM 1861). 